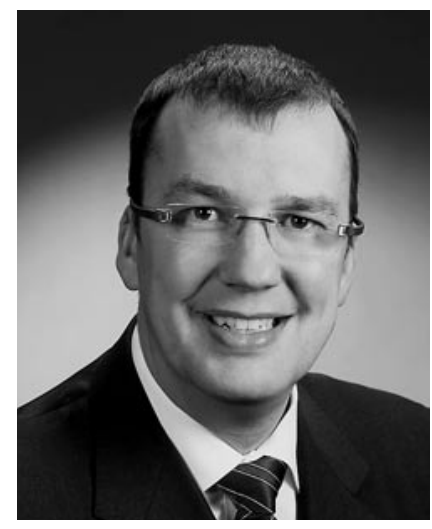

Utz Schäffer

\section{Ethische Fragestellungen sind auch im Controlling relevant!}

\section{Liebe Leser,}

im 53. Jahrgang der Zeitschrift für Controlling und Management muss konstatiert werden: Mit dem Thema Controlling und Ethik hat sich bislang noch kein Schwerpunktheft befasst. Das mag verwundern, umfasst doch so gut wie jedes amerikanische Accounting-Lehrbuch einige Seiten zu diesem Themenfeld. Und die grundsätzliche Relevanz ethischer Fragestellungen wird - spätestens nach einem Blick in die Tageszeitung - auch kaum ein Controller abstreiten wollen.

Jedoch findet sich häufig die Einstellung, dass Controlling wenig mit Ethik zu tun hat. Controllerbereiche schaffen demnach betriebswirtschaftliche Transparenz und stellen sicher, dass die Mittel dem Zweck der Ergebnis- oder Shareholdevaluemaximierung dienen. Mit ethischen Fragestellungen müssen sich dann wohl die Anderen - das Management - herumschlagen. Daneben gibt es oft eine gewisse Reaktanz gegenüber ethischen Themen: Man solle doch bitte moralisch handeln (oder nicht), aber keine stundenlangen Diskussionen führen oder seitenlange Abhandlungen zu dem Thema verfassen! Zu oft schon scheinen Begriffe wie Ethik und Nachhaltigkeit in Geschäftsberichten und Hochglanzbroschüren zu Legitimations- und Marketingzwecken mißbraucht worden zu sein. Aber wollen wir uns als Controller wirklich heraushalten, wenn es um die Gestaltung des richtigen Anreizsystems geht? Und spielen ethische Fragestellungen dabei wirklich keine Rolle? Ist es für uns Controller wirklich nicht relevant, darüber zu streiten, wie Umweltschutz, Nachhaltigkeit, gesellschaftlicher Impact und ähnliche Aspekte am besten gemessen werden können? Die Liste mit Fragen ließe sich fortsetzen.

Im vorliegenden Heft nehmen wir uns dem Spannungsfeld von Ethik und Controlling an. Am Anfang steht der Dialog. Prof. Dr. Andreas Suchanek vertritt im Interview mit Prof. Dr. Bernhard Hirsch unter anderem die These, dass es keinen betriebswirtschaftlichen Bereich gibt, in dem ethische Fragestellungen keine Relevanz besitzen. Und Prof. Dr. Volker Lingnau und ich diskutieren die Implikationen des Zusammenspiels von Ethik und Controlling auf die tägliche Controllerarbeit. Auf den Dialog folgt ein Plädoyer. Es freut mich als Herausgeber ganz besonders, dass es uns gelungen ist, Herrn Dr. Manfred Lautenschläger dafür zu gewinnen. Er fordert Mut zur Moral und argumentiert, dass dies in langfristiger Perspektive auch betriebswirtschaftlich rational ist. Praxisberichte schließen sich an: Zunächst berichten Dr. Dirk Reiche und Dr. Anna Herrhausen, wie bei der Allianz daran gearbeitet wird, den Nutzen gesellschaftlichen Engagements konkret zu messen. Im Anschluss befassen sich die Beiträge von Dieter Horst/Patrick Albrecht und Joachim Schlange mit wichtigen Aspekten der Corporate Responsibility: zum einen mit der Steuerung und Rechenschaftslegung "weicher" Erfolgsfaktoren, zum anderen mit der Corporate Responsibility Berichterstattung. Ein Transferbeitrag und eine Leseliste runden das Schwerpunktthema wie gewohnt ab. Nicht zuletzt möchte ich Sie noch auf die spannenden Beiträge von Prof. Dr. Wolfgang Becker und Patrick Ulrich zum Controlling im Mittelstand und von Prof. Dr. Matthias Meyer, Dr. Hendrik Schlüter und Dr. Carsten Sieber zur Rolle des Zentralcontrollings in der Investitionsplanung und -kontrolle hinweisen.

Viel Spaß bei der Lektüre wünscht Ihnen

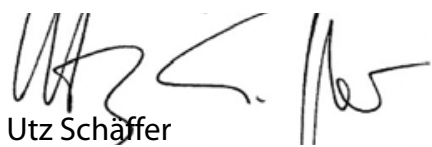

